\title{
Traditional Biocidal Replacement Viability of Microcrystalline Silver Chloride
}

\author{
Hemminger $\mathbf{Z}^{1}$, Walsh $\mathbf{P}^{2}$, Curtis $\mathbf{R}^{3}$ and Picardo $\mathbf{K}^{1 *}$ \\ ${ }^{1}$ Department of Biology, St. John Fisher College 3690 East Ave Rochester, NY, USA \\ ${ }^{2}$ Cerion, Rochester, NY, USA \\ ${ }^{3}$ Anovotek, Webster, NY, USA
}

\begin{abstract}
The antimicrobial effects of silver ions and silver chloride nanoparticles have been well established while the efficacy of microcrystalline silver chloride has been less studied. Certex-AM, a microcrystalline silver chloride product produced by Cerion, Rochester, NY, was tested for its antimicrobial properties as a possible replacement for traditional biocidal techniques used in water cooling towers. The minimum inhibitory concentration (MIC) of the compound was determined using a microtiter broth assay. The compound was found to have inhibitory effects on bacterial growth for all tested organisms at concentrations greater than $9 \mathrm{ppm}$. Additional testing simulating a water cooling system showed the effectiveness of reducing an established wild population at concentrations of $10 \mathrm{ppm}$ of the microcrystalline silver chloride. Certex-AM was found to be a promising replacement for traditional biocides as well as for other applications. Introduction of effective antimicrobial compounds such as this could reduce the pathogenic risk to humans associated with water cooling towers.
\end{abstract}

Keywords: MIC; Silver chloride; Silver nanoparticles; Microcrystalline silver chloride; Biocide; Antimicrobial

\section{Introduction}

The antimicrobial activity of silver and silver nanoparticles have been known since before the $18^{\text {th }}$ century $[1,2]$. Although the antimicrobial mechanisms are still being developed, many targets for silver interference have been proposed or established. One major target is the cellular membrane where the size of the particles compared to microbes results in a large surface area contact between the two causing physical interference as well as molecular [1]. It is theorized that this might assist in the ionic silvers ability to penetrate the membrane as well as interfere with respiration [2].

In addition, silver has been shown to have affinity for thiol groups, especially the amino acid cysteine and amino groups [1]. This binding would disrupt disulfide bridges and affect the polarity of the peptide. The structure of the protein would then change to stabilize the new charge and the functionality of the protein would be reduced or lost. In microbial membrane proteins, it is suggested that silver inhibits cellular respiration by uncoupling oxidative phosphorylation [1]. Beyond thiol group binding, the increase in reactive oxygen species have been observed as a result of silver particle exposure [3]. These reactive oxygen species, along with the silver ions, have also been shown to interact with protein and DNA resulting in denaturation [1]. As a result of the diverse antimicrobial mechanisms of silver there are fewer instances of silver resistance compared to antimicrobials with more specific targets. Higher level eukaryotes, specifically mammals, utilize antioxidant defense as well as metallothonein binding to reduce the toxicity [4]. The membrane interference effect of silver is also reduced since eukaryotes perform cellular respiration in the mitochondrial membranes compared to the outer membranes of prokaryotes. The efficacy of silver particles on microbial life, as well as the reduction in risk to mammalian life, suggests the role that silver could and does have as an antimicrobial agent.

The solubility properties of silver chloride and the bonding ability of silver ions are ideal for antimicrobial applications. Silver chloride in water is relatively insoluble with an ionic concentration of less than $2 \mathrm{ppm}$. Ionic silver in the presence of microorganisms will form coordinate bonds with the lone pairs of nitrogen atoms present in the organic molecules of proteins, nucleic acids, and other nitrogen containing species often inhibiting them. This coordinate bonding reduces the concentration of ionic silver and shifts the equilibrium towards dissociation. The stability of the salt in the absence of organisms along with the increase in activity in the presence of organisms are ideal properties that aid in the antimicrobial applications of silver particles. One possible application of silver particles that would benefit from the physical properties is in water cooling systems.

Water cooling systems are used to remove heat from a facility through the process of evaporation. Heat is transferred from the facility to the water which is typically sprayed over a matrix. The combination of air circulation and surface area evaporate some of the water resulting in an overall temperature decrease in the system. Water cooling systems are present in everything from household air conditioners to hospital and industrial cooling systems. Conditions present in many of these systems are ideal for microorganism colonization. These organisms cause damage to the systems reducing efficiency [5]. Often these organisms are human pathogens and can pose a health risk. Currently a variety of antimicrobial treatments exist to reduce the microbial load of the system. These treatments include oxidizing and non-oxidizing methods, but halogens are most widely used [6]. These treatments are often insufficient for controlling the biological load present and pose additional health risks associated with ingestion, inhalation, and skin contact. Certex-AM a $\sim 125 \mathrm{~nm}$ cubic microcrystalline silver chloride

*Corresponding author: Kristin Picardo, Department of Biology, St. John Fisher College 3690 East Ave Rochester, NY, USA, Tel: 5858993802; E-mail: kpicardo@sjfc.edu

Received: February 28, 2017; Accepted: March 16, 2017; Published: March 21 2017

Citation: Hemminger Z, Walsh P, Curtis R, Picardo K (2017) Traditional Biocida Replacement Viability of Microcrystalline Silver Chloride. J Nanomed Nanotechnol 8: 431. doi: 10.4172/2157-7439.1000431

Copyright: (c) 2017 Hemminger Z, et al. This is an open-access article distributed under the terms of the Creative Commons Attribution License, which permits unrestricted use, distribution, and reproduction in any medium, provided the original author and source are credited. 
product produced by Cerion, Rochester, NY was tested as a possible replacement to these dated biocidal techniques.

The majority of work regarding the antimicrobial properties of silver has occurred on ionic silver or silver particles below $100 \mathrm{~nm}$. Increasing the size of the particles could alter some of the antimicrobial properties. With increased size also comes increased surface area that could interrupt a larger region of the plasma membrane. Larger size also increases the number of ions present to disrupt cellular functions. Over time as the particle releases ions the surface area will decrease and the ion concentration will drop. Having a larger particle would theoretically extend the time that the particle remains effective. Antimicrobial efficacy testing was performed using Certex-AM in the context of a water cooling tower to test these claims.

\section{Materials and Methods}

\section{Bacterial strain information}

Organisms stored at $-80^{\circ} \mathrm{C}$ in Tryptic Soy Broth (TSB) containing $50 \%$ Glycerol and recovered in at TSB at $37^{\circ} \mathrm{C}$ with agitation (Table 1).

\section{Minimum inhibitory concentration determination}

Organisms were cultured on Mueller Hinton II (MHII) Agar plates (Becton, Dickinson and Company, Sparks, MD) at $37^{\circ} \mathrm{C}$ for 16 hours with agitation. Cultures were prepared by inoculating 4-5 colonies of pure culture in MHII broth (Becton, Dickinson and Company, Sparks, $\mathrm{MD}$ ) and incubating at $37^{\circ} \mathrm{C}$ for $18-22$ hours. Cultures were then diluted with MHII Broth until an $\mathrm{OD}_{600}$ of 0.16 was reached. Antimicrobial solutions were created at twice the desired concentration to be tested to compensate for a dilution when the culture was added. $100 \mu \mathrm{L}$ of the antimicrobial solution and $100 \mu \mathrm{L}$ of the bacterial cultures were added in triplicate to 96 well plates (96 well flat bottom non treated polystyrene assay plates with low evaporation lid Costar, Kennebunk, ME). Control wells with sterile MHII Broth were prepared for each antimicrobial concentration to ensure sterility. $\mathrm{OD}_{600}$ readings were taken immediately after inoculation. Plates were incubated at $37^{\circ} \mathrm{C}$ for 18-22 hours and the $\mathrm{OD}_{600}$ was measured. The point at which the difference between the $\mathrm{OD}_{600}$ prior to incubation and after incubation was at or below zero was determined to be the Minimum Inhibitory Concentration (Table 1).

\begin{tabular}{|l|c|c|}
\hline MIC Values \\
\hline Organism & ATCC & MIC (ppm) \\
\hline Bacillus cereus & 13061 & $6-9$ \\
\hline Citrobacter freundii & 43864 & $3-6$ \\
\hline Enterobacter aerogenes & 13048 & $3-6$ \\
\hline Enterococcus faecalis & 19433 & $3-6$ \\
\hline Klebsiella pneumoniae & 13883 & $3-6$ \\
\hline Pseudomonas aeruginosa & PAO1 & $3-6$ \\
\hline Staphylococcus aureus & 29213 & $6-9$ \\
\hline Staphylococcus epidermidis & 14990 & $6-9$ \\
\hline Shigella sonnei & 25931 & $3-6$ \\
\hline Salmonella typhimurium & 14028 & $6-9$ \\
\hline Staphylococcus saprophyticus & 15305 & $3-6$ \\
\hline Streptococcus mutans & 25175 & $6-9$ \\
\hline
\end{tabular}

Table 1: Minimum inhibitory concentrations: the Minimum Inhibitory Concentration (MIC) was determined to be the point at which exposure to a concentration of antimicrobial for 24 hours results in no growth. Growth was determined by $\mathrm{OD}_{600}$ readings. The point at which the $O D_{600}$ reading after the exposure was the same as prior to exposure was determined to be the MIC. The range provided is the highest concentration of the antimicrobial in which growth occurred $\left(\mathrm{OD}_{600}\right.$ increased) and the lowest concentration in which growth was inhibited $\left(\mathrm{OD}_{600}\right.$ stayed the same or decreased). The MIC was determined to be between these values.

\section{Microbial ecology identification}

Water samples taken from a local water cooling tower, Rochester, NY were filtered through $125 \mathrm{~nm}$ filters. Resulting filters were incubated on R2A Agar (Becton, Dickinson and Company, Sparks, MD) for $24-48$ hours at $25^{\circ} \mathrm{C}$. Resulting growth was subcultured on R2A Agar until pure samples were obtained. Cultures were prepared by inoculating a laboratory colony in Luria Broth (LB) (Becton, Dickinson and Company, Sparks, MD) and incubating at $37^{\circ} \mathrm{C}$ for $12-$ 16 hours. Bacterial cultures were pelleted at 14,000 RPM for 3 min and the resulting supernatant was decanted. Cells were lysed through the addition of $4 \mu \mathrm{L}$ RNase A, $20 \mu \mathrm{L}$ Proteinase $\mathrm{K}$, and $200 \mu \mathrm{L}$ Lysis Buffer [2\% Triton, $1 \%$ SDS, $100 \mathrm{mM} \mathrm{NaCl}, 1 \mathrm{mM} \mathrm{Na}_{2}$ EDTA]. The resulting solution was mixed thoroughly and incubated at $37^{\circ} \mathrm{C}$ for $30 \mathrm{~min}$ with agitation and at $95^{\circ} \mathrm{C}$ for $10 \mathrm{~min}$. To the tube, $1000 \mu \mathrm{L}-20^{\circ} \mathrm{C} \mathrm{EtOH}$ was added and the resulting solution was cooled at $-20^{\circ} \mathrm{C}$ for $30 \mathrm{~min}$. The solution was added to a Qiagen QIAamp Mini Spin Column with a 2 $\mathrm{mL}$ centrifuge tube to collect flow through. The column was centrifuged at 8,000 RMP for 1 minute and the flow through was discarded. To the column, $600 \mu \mathrm{L}$ of Omega DNA Wash Buffer was added and centrifuged at 8,000 RPM for a minute. This was repeated twice. The column was centrifuged at 14,000 RPM for 5 min to dry the column. The collection tube was replaced and $50 \mu \mathrm{L}$ elution buffer was added. The column was incubated at $37^{\circ} \mathrm{C}$ for $15 \mathrm{~min}$ and centrifuged at $8,000 \mathrm{RPM}$ for a minute. The DNA extraction product was collected and stored at $-20^{\circ} \mathrm{C}$. To a PCR tube, $46 \mu \mathrm{L}$ of a master mix [ $1392 \mu \mathrm{L}$ Sterile deionized water, $200 \mu \mathrm{L}$ GenScript 10x PCR Buffer, $40 \mu \mathrm{L} 10 \mu \mathrm{M}$ GenScript dNTPs, $100 \mu \mathrm{L} 10 \mu \mathrm{M}$ Forward Primer Integrated DNA Technologies (IDT) SSU-bact-27f (5'-AGA GTT TGA TCM TGG CTC AG-3'), 100 $\mu \mathrm{L} 10 \mu \mathrm{M}$ Reverse Primer Integrated DNA Technologies (IDT) SSUbact-519r (5'-GWA TTA CCG CGG CKG CTG-3'), and $8 \mu \mathrm{L} 5 \mathrm{U} / \mu \mathrm{L}$ GenScript Taq DNA Polymerase] and $4 \mu \mathrm{L}$ of DNA extraction product were added and a routine thermocycle reaction was performed. The PCR product was stored at $-20^{\circ} \mathrm{C}$. The PCR Product was transferred to a $1.5 \mathrm{~mL}$ centrifuge tube containing $300 \mu \mathrm{L}$ Omega Citrate Phosphate Buffer. The solution was transferred to a HIBind DNA Column and a 2 $\mathrm{mL}$ centrifuge tube was attached. The column was centrifuged at 14,000 RPM for a min. To the column, $700 \mu \mathrm{L}$ DNA Wash Buffer was added and centrifuged at 14,000 RPM for a min. the filtrate was discarded. The process was repeated. The column was centrifuged for 14,000 RPM for a min to dry the column. To the column, $30 \mu \mathrm{L}$ of Elution Buffer was added and the collection tube was replaced. The column was incubated at $37^{\circ} \mathrm{C}$ for $30 \mathrm{~min}$ and centrifuged for $14,000 \mathrm{RPM}$ for $3 \mathrm{~min}$. The filtrate was collected and stored at $-20^{\circ} \mathrm{C}$. Purified PCR product as well as Forward and Reverse Primers were sent to ACTG for Sequencing. Sequences were compared to 16s Ribosomal DNA Sequences of known species in Blast database. Sequence results are recorded in Figure 1.

\section{Neutralization study}

Bacterial Cultures of Staphylococcus aureus ATCC 29213, Klebsiella pneumoniae ATCC 13833, and Pseudomonas aeruginosa PAO1 were cultured in TSB from pure isolated colonies grown on TSA and incubated at $37^{\circ} \mathrm{C}$ for $18-22$ hours. Cultures were pelleted at 13,000 RPM for 4 min and the supernatant was decanted. Pellets were washed in 1XPBS and pelleted at 13,000 RPM for $4 \mathrm{~min}$. The supernatant was decanted and the pellets were resuspended in 1XPBS to an $\mathrm{OD}_{600}$ of 0.14 . Bacterial solutions were serial diluted by a factor of 4 in 1XPBS To $900 \mu \mathrm{L}$ of DE Neutralizing Broth (DEB) (Becton, Dickinson and Company, Sparks, MD), $100 \mu \mathrm{L}$ of 100 ppm antimicrobial was added and allowed to neutralize for $10 \mathrm{~min}$. To the neutralized antimicrobial, $100 \mu \mathrm{L}$ of the bacterial solution was added and $100 \mu \mathrm{L}$ was spread plated 


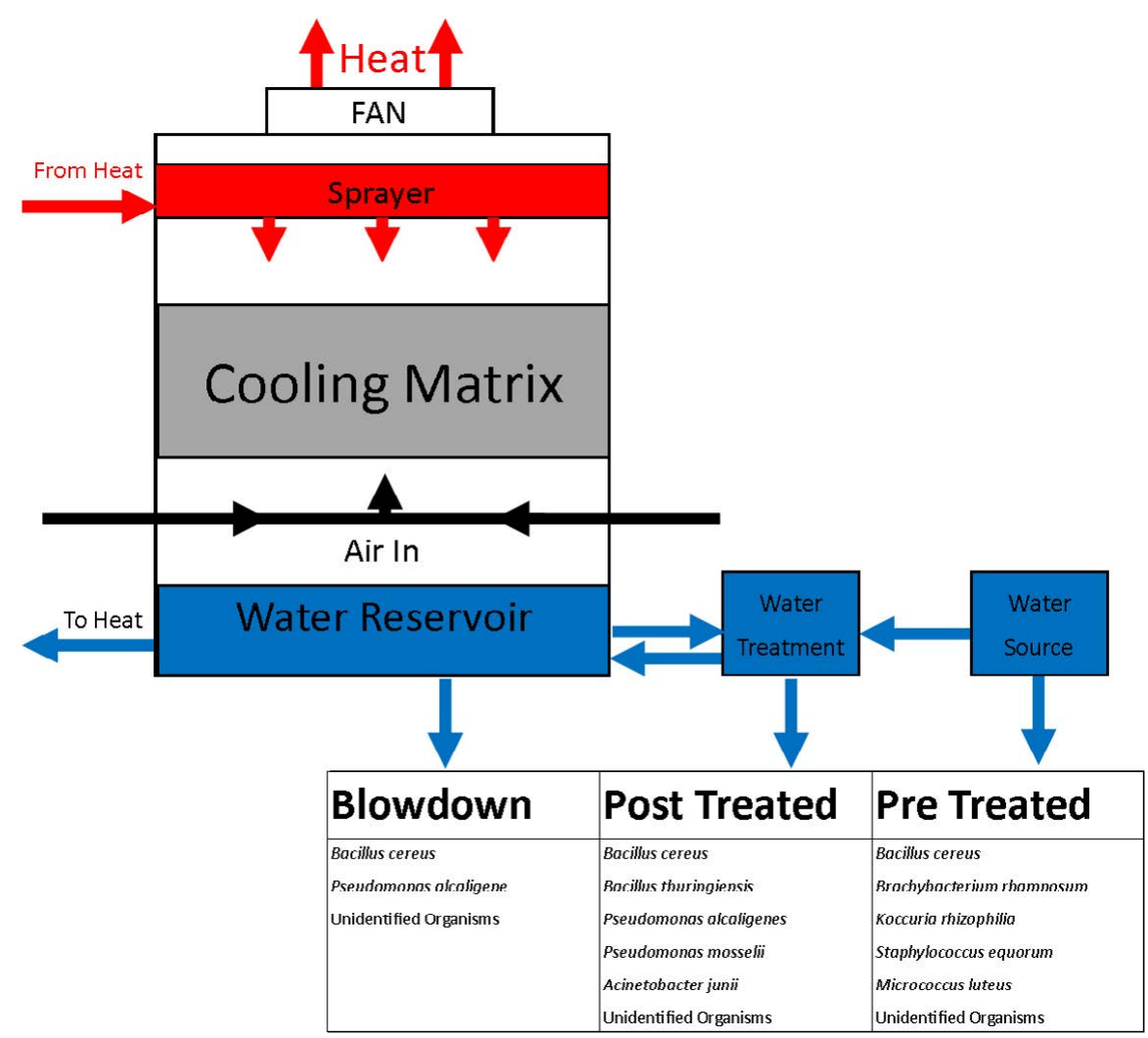

Figure 1: Microbial ecology determination: Organisms extracted from a local water tower, Rochester NY were identified by $16 S$ Ribosomal DNA Sequencing and compared to Blast database. Isolation locations of the identified organisms are shown above on an over simplified diagram of the water cooling tower.

on R2A. Plates were incubated at $25^{\circ} \mathrm{C}$ for 16 hours and colonies were counted and compared to bacterial solutions exposed to DEB without the antimicrobial (Figure 2).

\section{Laboratory organism dosage study}

Bacterial cultures were prepared in TSB from isolated colonies and incubated at $37^{\circ} \mathrm{C}$ for $18-22$ hours. Cultures were pelleted at 13,000 RPM for 4 min and the supernatant was decanted. Pellets were washed in 1XPBS and pelleted at 13,000 RPM for $4 \mathrm{~min}$. The pellets were resuspended in $10 \%$ TSB to an $\mathrm{OD}_{600}$ of 0.14 . In a $250 \mathrm{~mL}$ Flask, $100 \mathrm{~mL}$ $10 \%$ TSB was added with $1 \mathrm{~mL}$ of the bacterial culture. Antimicrobial was added to make a final concentration of $10 \mathrm{ppm}$. Flasks were incubated at $25^{\circ} \mathrm{C}$ at $110 \mathrm{RPM}$ for a week. Samples were taken at 0,1 , $2,3,5,24,48,120$ hours. To $900 \mu \mathrm{L} \mathrm{DEB}, 100 \mu \mathrm{L}$ of the samples were added and the antimicrobial was allowed to neutralize for 10 minutes. On R2A plates, $100 \mu \mathrm{L}$ of the neutralized samples were plated and incubated at $25^{\circ} \mathrm{C}$ for 16 hours. Colonies were counted and compared to cultures not exposed to antimicrobial (Figure 3).

\section{Multi-organism dosage study}

In a $2 \mathrm{~L}$ Flask, $1 \mathrm{~L}$ of Water Samples collected from a local water cooling tower, Rochester NY was added. Antimicrobial was added to make a final concentration of $10 \mathrm{ppm}$. Flasks were incubated at $25^{\circ} \mathrm{C}$ at 110 RPM for a week. Samples were taken at 0, 1, 2, 3, 5, 24, 48, 120 hours. To $900 \mu \mathrm{L} \mathrm{DEB}, 100 \mu \mathrm{L}$ of the samples were added and the antimicrobial was allowed to neutralize for 10 minutes. On R2A plates, $100 \mu \mathrm{L}$ of the neutralized samples were plated and incubated at $25^{\circ} \mathrm{C}$ for 16 hours. Colonies were counted and compared to cultures not exposed to antimicrobial (Figure 4).

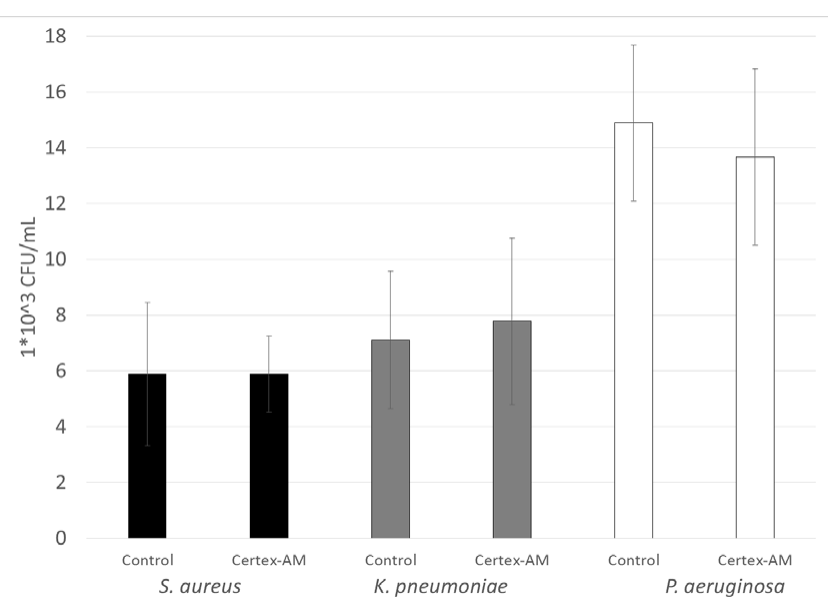

Figure 2: Neutralization Study: Antimicrobial was neutralized in DE Neutralizing Broth (DEB) and the survivability of the solution is shown above. The number of colony forming units (CFU) present after exposure to the neutralized antimicrobial is shown above to be insignificant to the number of CFU present after exposure to DEB that had no antimicrobial present.

\section{Results}

Minimum Inhibitory Concentration Determination- The microcrystalline silver chloride was found to have inhibitory properties for concentrations greater than $6 \mathrm{ppm}$ for most organisms and $9 \mathrm{ppm}$ for all organisms (Table 1).

Microbial Ecology Identification-Organisms present in a local 
water cooling tower at different locations were isolated and identified through 16S Ribosomal DNA sequencing and compared to Blast database (Figure 1).

Neutralization Study-The survivability of organisms in DEB was found to be the same as the survivability of organisms in DEB with Certex-AM. DEB was determined to be an effective neutralizer of the crystalline silver chloride (Figure 2).

Laboratory Organism Dosage Study-A population of Pseudomonas aeruginosa, a common water born bacteria, of $1^{\star} 10^{5} \mathrm{CFU} / \mathrm{mL}$ was reduced to below the limit of detection of $100 \mathrm{CFU} / \mathrm{mL}$ within 5 hours when exposed to $10 \mathrm{ppm}$ of Certex-AM (Figure 3).

Multi-Organism Dosage Study-A population of bacteria from

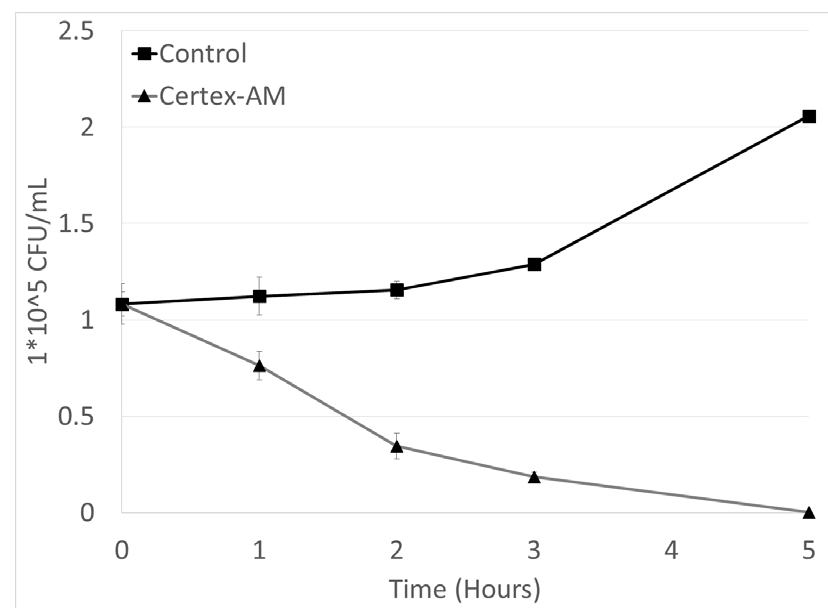

Figure 3: Laboratory Organism Water Cooling Tower Dosage Simulation: $P$. aerigunosa was seeded in $10 \%$ TSB and exposed to $10 \mathrm{ppm}$ Certex-AM Samples taken were neutralized in DE Neutralizing Broth (DEB) for 10 minutes to deactivate the antimicrobial and allow the Colony forming units (CFU) to grow on the R2A Plates. No CFU were present in the Certex-AM Dosed samples after 5 hours. Samples continued to have no CFU up to 120 hours.

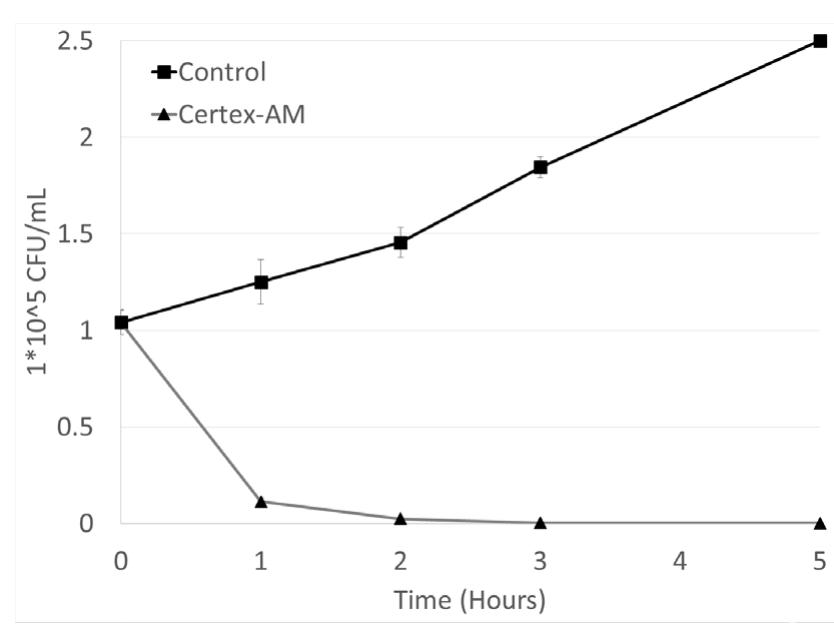

Figure 4: Multi-Organism Water Cooling Tower Dosage Simulation: water Samples were exposed to $10 \mathrm{ppm}$ Certex-AM. Samples taken were neutralized in DE Neutralizing Broth (DEB) for 10 minutes to deactivate the antimicrobial and allow the Colony forming units (CFU) to grow on the R2A Plates. No CFU were present in the Certex-AM Dosed samples after 2 hours. Samples continued to have no CFU up to 120 hours. water samples taken from a local water cooling tower were exposed to $10 \mathrm{ppm}$ of the compound reducing its initial concentration of $1^{\star} 10^{5}$ $\mathrm{CFU} / \mathrm{mL}$ to below our limit of detection of $100 \mathrm{CFU} / \mathrm{mL}$ within 2 hours (Figure 4).

\section{Discussion}

In order to test the efficacy of an antimicrobial it is necessary to be able to accurately measure the microbial population present at any time during antimicrobial exposure. In order to culture samples taken during this antimicrobial exposure a neutralizing agent must be used. Neutralizing agents inactivate the antimicrobial to allow accurate exposure times. The neutralizing agent DEB was tested for its effectiveness at neutralizing Certex-AM and was found to be effective after 10 minutes when 1 part sample was mixed with 9 parts DEB. At this concentration and time it was found that DEB neutralized CertexAM to the point that organisms exposed to DEB survived statistically the same as organisms exposed to DEB neutralized Certex-AM.

In an effort to determine a working dose for the antimicrobial the MIC was determined for a wide range of laboratory organisms that the antimicrobial could potentially be exposed to. Concentrations above 9 ppm were consistently able to prevent growth of all organisms tested. A working dose of $10 \mathrm{ppm}$ was used for all successive trials.

The organisms present in a true antimicrobial exposure present won't be laboratory strains but wild strains. In order to determine the effectiveness of the working dose on wild organisms it was necessary to develop a simulated dosage study. A single laboratory organism was used to develop the efficacy of the technique. Water cooling tower water collected from the blowdown was run through the developed dosage study at the working dose of $10 \mathrm{ppm}$. The antimicrobial was effective at reducing a population of $\sim 1 \mathrm{E}+05 \mathrm{CFU} / \mathrm{mL}$ to below 100 $\mathrm{CFU} / \mathrm{mL}$ within 5 hours. The sample remained below this level beyond 5 days when sample acquisition was terminated.

Microbial identification of the water sample from various locations of the water cooling tower was performed. Presence of organisms in the water source as well as immediately after traditional water treatment and in the blowdown of the tower shows that organisms present in the water source for the tower were surviving the traditional antimicrobial treatment. These organisms which survived the traditional treatment were effectively controlled by a concentration of $10 \mathrm{ppm}$ of Certex-AM.

Results observed during experimentation are consistent with similar published studies [2,7,8]. Deviations from observed MIC between studies results from difference in particle size as well as experimental methods [7]. Studies also show the importance of particulate metal oxides and metal salts as antimicrobial agents towards eukaryotic as well as prokaryotic microorganisms $[8,9]$. The efficacy of this class of antimicrobial agents as well as the range of applications highlight the importance of understanding and developing technologies to control microbial populations.

The problem with most antimicrobial applications is the requirement for constant dosing of the system to maintain antimicrobial control. Development of technologies that could reduce dosing would benefit these systems as well as other applications. Silver particles have characteristics, such as self-dosing, which make it a suitable candidate to reduce this problem. This self-dosing characteristic comes from the structure of the product. The majority of the silver present is bound within the crystalline structure and is not participating in the antimicrobial activity. The only active silver comes from the surface of the crystals as well as ionic silver that is dispersed from the surface. As 
Citation: Hemminger Z, Walsh P, Curtis R, Picardo K (2017) Traditional Biocidal Replacement Viability of Microcrystalline Silver Chloride. J Nanomed Nanotechnol 8: 431. doi: 10.4172/2157-7439.1000431

the concentration of active silver decreases more silver is released to replenish it. This self-dosing capability allows us to treat samples with a larger dose than necessary without the risks associated with higher doses. The ionic silver concentration is the critical determinant of if the antimicrobial level is being reached as well as if the human health level is reached. The silver bound within the matrix is essentially inert and doesn't participate in either process until released. The health effects associated with insoluble silver such as microcrystalline silver chloride poses limited to no health affects at the low concentrations needed to control microbial life [10]. This allows the larger doses without the human health implications. This self-dosing property would reduce the number of doses that an operator would need to perform while still maintaining control of the biological population present. This property is useful for the water cooling tower industry, but also has uses in other applications. Certex-AM specifically has advantages that other smaller particles lack. Compared to a $100 \mathrm{~nm}$ size cubic particle a $125 \mathrm{~nm}$ cubic particle would have over 50\% larger surface area and each particle would contain almost twice as much silver. This increases the interference of membranes as well as provides a larger storage of silver to reduce the need to dose.

Some situations make repeated dosing an inconvenience while other situations the idea of repeated dosing is impossible. An example of this would be in textiles. In textiles the antimicrobial is bound to the fibers of the textile. As these compounds are used up the concentration falls below the level necessary to control bacterial growth. Application of additional doses to the textiles is not likely. Instead the use of self-dosing antimicrobials would allow the concentration of the antimicrobial to stay within the buffer region above the inhibitory concentration and below the human health concentration. Technologies such as this have a broad range of applications from antimicrobial clothes and linens to carpets and paints. Active control of the biological populations present in items that humans come in contact with every day could greatly reduce the risk associated of uncontrolled bacterial populations to human health.

\section{References}

1. Duran N, Duran M, De Jesus MB, Seabra AB, Favaro WJ, et al. (2016) Silver nanoparticles: A new view on mechanistic aspects on antimicrobial activity. Nanomedicine 3: 789-99.

2. Rai MK, Deshmukh SD, Ingle AP, Gade AK (2012) Silver Nanoparticles: the powerful nanoweapon against multidrug-resistant bacteria. Journal of Applied Microbiology 112: 841-852.

3. Kim S, Ryu DY (2013) Silver nanoparticle-induced oxidative stress, genotoxicity and apoptosis in cultured cells and animal tissues. Journal of Applied Toxicology 33(2): 78-89.

4. Zhang H, Wang X, Wang M, Li L, Chang CH, et al. (2015) Mammalian cells exhibit a range of sensitivities to silver nanoparticles that are partially explicable by variations in antioxidant defense and metallothionein expression. Small 11 : 3797-3805.

5. Kip N, Veen J (2015) The dual role of microbes in corrosion. ISME 9: 542-551.

6. Rose LJ, Rice EW (2014) Inactivation of bacterial biothreat agents in water, a review. J Water Health 12: 618-633.

7. Petrus EM, Tinakumari S, Chai S, Ubong LC, Tunung R, et al. (2011) A study on the minimum inhibitory concentration and minimum bactericidal concentration of Nano Colloidal Silver on food-borne pathogens. International Food Research Journal 15: 55-66.

8. Raghunath A, Perumal E (2017) Metal oxide nanoparticles as antimicrobial agents: a promise for the future. International Journal of Antimicrobial Agents 49: 137-152.

9. Singh R, Nawale LU, Arkile M, Shedbalkar UU, Wadhwani S, et al. (2015) Chemical and biological metal nanoparticles as antimycobacterial agents: A comparative study. International Journal of Antimicrobial Agents 46: 183-188.

10. Drake PL, Hazelwood KJ (2005) Exposure-Related Health Effects of Silver and Silver Compounds: A Review. Annals of Occupational Hygiene 49: 575-585. 